PROCEEDINGS OF THE

AMERICAN MATHEMATICAL SOCIETY

Volume 135, Number 1, January 2007, Pages 41-45

S 0002-9939(06)08628-X

Article electronically published on June 30, 2006

\title{
IRREDUCIBLE CHARACTERS WHICH ARE ZERO ON ONLY ONE CONJUGACY CLASS
}

\author{
JOHN D. DIXON AND A. RAHNAMAI BARGHI
}

(Communicated by Jonathan I. Hall)

\begin{abstract}
Suppose that $G$ is a finite solvable group which has an irreducible character $\chi$ which vanishes on exactly one conjugacy class. Then we show that $G$ has a homomorphic image which is a nontrivial 2-transitive permutation group. The latter groups have been classified by Huppert. We can also say more about the structure of $G$ depending on whether $\chi$ is primitive or not.
\end{abstract}

\section{INTRODUCTION}

Let $\chi$ be an irreducible character of a finite group $G$. A well-known theorem of Burnside [9, page 40] shows that when $\chi$ is nonlinear it takes the value 0 on at least one conjugacy class of $G$. Groups having an irreducible character that vanishes on exactly one class were studied by Zhmud' in [1] (see also [1]). Chillag [2, Lemma $2.4]$ has proved that if the restriction of $\chi$ to the derived group $G^{\prime}$ is reducible and $\chi$ vanishes on exactly one class of $G$, then $G$ is a Frobenius group with a complement of order 2 and an abelian odd-order kernel.

Our purpose in this paper is to show that, if an irreducible character $\chi$ of a finite solvable group $G$ vanishes on exactly one conjugacy class, then $G$ has a homomorphic image which is a nontrivial 2-transitive permutation group. The latter groups have been classified by Huppert: they have degree $p^{d}$ where $p$ is prime, and are subgroups of the extended affine group $A \Gamma L\left(1, p^{d}\right)$ except for six exceptional degrees (see Remark 8 below). We can also say more about the structure of $G$ depending on whether $\chi$ is primitive or not.

\section{MAin Results}

We shall initially assume that our character is faithful, and make the following assumptions:

(*) $G$ is a finite group with a faithful irreducible character $\chi$ which is 0 on only one class which we denote by $\mathcal{C}$. Furthermore, $G$ has a chief factor $K / L$ which is an elementary abelian $p$-group of order $p^{d}$ such that the restriction $\chi_{K}$ is irreducible, but $\chi_{L}$ is not.

Since $\chi$ must be nonlinear, the latter condition clearly holds whenever $G$ is solvable, but for the present we shall not assume solvability.

Received by the editors August 4, 2005.

2000 Mathematics Subject Classification. Primary 20C15, 20D10, 20 B20.

(C)2006 American Mathematical Society Reverts to public domain 28 years from publication 
Proposition 1. Suppose (*) holds. Then $\mathcal{C}=K \backslash L, K=\langle\mathcal{C}\rangle$ and $L$ is equal to $L_{0}:=\{u \in G \mid u \mathcal{C}=\mathcal{C}\}$. In particular, $\mathcal{C}$ consists of p-elements (since $L$ does not contain a Sylow p-subgroup of K). Moreover, either:

(i) $d$ is even, $\chi_{L}=p^{d / 2} \phi$ for some nontrivial linear character $\phi$ of $L$, and $L=Z(G)$; or

(ii) $\chi_{L}=\phi_{1}+\ldots+\phi_{p^{d}}$ is the sum of $p^{d}$ distinct $G$-conjugate irreducible characters of $L$, and so $\chi$ is imprimitive and $p^{d} \mid \chi(1)$.

Proof. Since $\chi_{K}$ is irreducible, the theorem of Burnside quoted above shows that $\mathcal{C} \subseteq K$. On the other hand, since $\chi_{L}$ is reducible, 1, Theorem 21.1] shows that $\mathcal{C} \cap L=\emptyset$. Hence $\mathcal{C} \subseteq K \backslash L$.

Now since $K / L$ is an abelian chief factor, and $\chi_{K}$ is irreducible, it follows from 9. (6.18)] that either (i) $d$ is even, $\chi_{L}=p^{d / 2} \phi$ for some $\phi \in \operatorname{Irr}(L)$; or (ii) $\chi_{L}=$ $\phi_{1}+\ldots+\phi_{p^{d}}$ is the sum of $p^{d}$ distinct $G$-conjugate irreducible characters $\phi_{i}$. We shall consider these two cases separately.

In case (i) we note that, since $\mathcal{C} \cap L=\emptyset$, the irreducible character $\phi$ does not take the value 0. Thus Burnside's theorem implies that $\phi(1)=1$. This implies that if $\rho$ is a representation affording $\chi$, then $\rho$ is scalar on $L$. Since $\chi$ is assumed to be faithful, $L$ is contained in the centre $Z(G)$ of $G$. On the other hand, for each $z \in Z(G), \rho(z)$ is a scalar of the form $\zeta 1$. Thus for each $x \in \mathcal{C}$ we have $\chi(z x)=\operatorname{trace} \rho(z x)=\operatorname{trace} \zeta \rho(x)=\zeta \chi(x)=0$ and so $z x \in \mathcal{C}$. Therefore $z=$ $(z x) x^{-1} \in\langle\mathcal{C}\rangle \leq K$ for all $z \in Z(G)$. This shows that $Z(G)$ is a normal subgroup of $G$ satisfying $L \leq Z(G) \leq K$. Since $K / L$ is a chief factor and $\chi_{K}$ is a nonlinear irreducible character of $K$, we conclude that $Z(G)=L$. Finally, since $K / Z(G)$ is abelian, 9, (2.30)] shows that $K \backslash L=\mathcal{C}$.

In case (ii) $\chi_{K}$ is an irreducible constituent of $\left(\phi_{1}\right)^{K}$ and so comparison of degrees shows that $\chi_{K}=\left(\phi_{1}\right)^{K}$. Thus $\chi_{K}$ is 0 everywhere outside of the normal subgroup $L$, and so $K \backslash L=\mathcal{C}$ in this case as well.

Finally since $|\mathcal{C} \cup\{1\}|>\frac{1}{2}|K|$, then $K=\langle\mathcal{C}\rangle$. Finally, it is easily seen that $L_{0}$ is a normal subgroup of $G$, and that $L_{0} \subseteq \mathcal{C C}^{-1}$ and so $L_{0} \leq K$. Since $\mathcal{C}=K \backslash L$ is a union of cosets of $L$, we see that $L \leq L_{0}$. On the other hand, $\mathcal{C} \nsubseteq L_{0}$ since $\mathcal{C}$ is not a subgroup. Therefore $L_{0} \triangleleft G$ and $L \leq L_{0}<K$; hence $L_{0}=L$ as claimed.

Corollary 2. Under the hypothesis (*) every normal subgroup $N$ of $G$ either contains $K$ (when $\chi_{N}$ is irreducible) or is contained in $L$ (when $\chi_{N}$ is reducible). In particular, $K / L$ is the unique chief factor such that $\chi_{K}$ is irreducible and $\chi_{L}$ is reducible and $K / L$ is the socle of $G / L$. Since $K$ has a nonlinear irreducible character, $K$ is not abelian and so $L \neq 1$.

Remark 3. Both cases (i) and (ii) in Proposition 1 can actually occur. The group $\mathrm{SL}(2,3)$ has three primitive characters of degree 2 which satisfy $(*)$ (case (i) with $|K|=8$ and $|L|=2$ for each character), and $S_{4}$ has an imprimitive character of degree 3 which satisfies (*) (case (ii) with $|K|=12$ and $|L|=4$ ).

Proposition 4. Suppose that the hypothesis (*) and case (i) of Proposition 1 hold. Then $L=Z(G)$ has order $p, K$ is an extra special p-group and $\chi$ is primitive.

Proof. Let $z \in L$. Then for any $x \in \mathcal{C}$ we have $z x \in \mathcal{C}$ and so $z x=y^{-1} x y$ for some $y \in G$. Since $K / L$ is an elementary abelian $p$-group, $z^{p} x^{p}=(z x)^{p}=y^{-1} x^{p} y=x^{p}$, and so $z^{p}=1$. Thus $L$ is of exponent $p$. Since $L=Z(G)$ is represented faithfully as a group of scalar matrices by a representation affording $\chi$, it follows that $L$ is 
cyclic and hence $|L|=p$. Because $K$ is nonabelian, $K^{\prime}=\Phi(K)=L=Z(K)$ and so $K$ is an extraspecial $p$-group.

We finally show that $\chi$ is primitive. Indeed, otherwise there is a maximal subgroup $H$ in $G$ and $\psi \in \operatorname{Irr}(H)$ such that $\chi=\psi^{G}$. The formula for an induced character shows that $\psi^{G}$ is 0 on each conjugacy class disjoint from $H$. As is well known every proper subgroup of a finite group is disjoint from some conjugacy class, and so we conclude that $\mathcal{C}$ is the unique class such that $\mathcal{C} \cap H=\emptyset$. By Proposition 1 this implies that $H \cap K \leq L$. Thus $K \not \leq H$, and so $G=H K$ by the maximality of $H$. Hence

$$
\chi(1)=\psi^{G}(1) \geq|G: H|=|K: H \cap K| \geq|K: L|=p^{d} .
$$

Since $\chi(1)=p^{d / 2}$, we obtain a contradiction. Thus $\chi$ is primitive.

Proposition 5. Suppose that the hypothesis (*) and case (ii) of Proposition 1 hold (so $\chi$ is imprimitive). Then there exists a subgroup $M$ of index $p^{d}$ in $G$ such that $\chi=\psi^{G}$ for some $\psi \in \operatorname{Irr}(M), G=M K$ and $M \cap K=L=\operatorname{core}_{G}(M)$.

Proof. As noted in the proof of Proposition $1 \chi_{L}$ is a sum of $p^{d}$ distinct irreducible constituents $\phi_{i}$. Because $\chi_{K}$ is irreducible, these constituents are $K$-conjugates (as well as $G$-conjugates). Let $M:=I_{G}\left(\phi_{1}\right)$ be the inertial subgroup fixing the constituent $\phi_{1}$. Then $|G: M|=p^{d}$ and $G=M K$ because $K$ acts transitively on the set of $\phi_{i}$. Clearly $L \leq M$. Since $|K: L|=p^{d}=|G: M|=|K: M \cap K|$, we conclude that $M \cap K=L$. On the other hand, since $\psi^{G}$ is 0 on any class which does not intersect $M$, the hypothesis on $\chi$ shows that $\mathcal{C}=G \backslash \bigcup_{y \in G} y^{-1} M y$. Now $u \in \operatorname{core}_{G}(M)=\bigcap_{y \in G} y^{-1} M y$ and $x \in \mathcal{C}$ implies that $u x$ does not lie in any $y^{-1} M y$, and hence $u x \in \mathcal{C}$. Thus with the notation of Proposition 1. $\operatorname{core}_{G}(M) \leq L_{0}=L$. Since $L$ is a normal subgroup contained in $M$, the reverse inequality is also true, and so $\operatorname{core}_{G}(M)=L$.

The proof of the next result requires a theorem of Isaacs [10, Theorem 2] which states:

Let $H$ be a finite group with centre $Z$ and let $K$ be a normal subgroup of $H$ with $Z=Z(K)$. Suppose that $H$ centralizes $K / Z$ and $|\operatorname{Hom}(K / Z, Z)| \leq|K / Z|$. Then $H / Z=K / Z \times C_{H}(K) / Z$.

Proposition 6. Under the hypothesis $(*)$ the centralizer $C_{G}(K / L)$ equals $K$.

Proof. If $\chi$ is primitive, then Proposition 4 shows that the hypotheses of Isaacs' theorem are satisfied for $H:=C_{G}(K / L)$ (the condition $|\operatorname{Hom}(K / Z, Z)| \leq|K / Z|$ is trivial since the irreducibility of $\chi_{H}$ implies that $Z$ is cyclic). Also, since $\chi_{K}$ is irreducible, $C_{G}(K)=Z(G)=L$, and so Isaacs' theorem shows that $H / L=$ $K / L \times C_{H}(K) / L=K / L$, as required.

If $\chi$ is imprimitive, then using the notation of Proposition 5 we can show that $M \cap H=L$, where $H:=C_{G}(K / L)$. Indeed, it is clear from Proposition 5 that $L \leq M \cap H$. To prove the reverse inequality suppose that $u \in M \cap H$. Then for each $x \in K$ we have $x u=y u x$ for some $y \in L$. Choose $i$ such that $\phi_{1}^{x}=\phi_{i}$. Then $\phi_{i}^{u}=\phi_{1}^{x u}=\phi_{1}^{y u x}=\phi_{1}^{x}=\phi_{i}$. Hence $u$ fixes $\phi_{1}^{x}$ and therefore lies in $x^{-1} M x$. Since this is true for all $x \in K$, it follows from Proposition 5 that $u \in \operatorname{core}_{G}(M)=L$. Thus $M \cap H=L$ as claimed. Finally $H=H \cap M K=(H \cap M) K=L K=K$, as required. 
Corollary 7. Under the hypothesis (*) G acts transitively by conjugation on the nontrivial elements of the vector space $K / L$, and the kernel of this action is $K$. Thus $G / K$ is isomorphic to a subgroup of $G L(d, p)$ which is transitive on the nonzero elements of the underlying vector space.

Remark 8. Huppert [8, Chapter XII Theorem 7.3] has classified all solvable subgroups $S$ of $G L(d, p)$ which are transitive on the nonzero vectors of the underlying vector space. Apart from six exceptional cases (where $p^{d}=3^{2}, 5^{2}, 7^{2}, 11^{2}, 23^{2}$ or $3^{4}$ ), the underlying vector space can be identified with the Galois field $G F\left(p^{d}\right)$ in such a way that $S$ is a subgroup of the group $\Gamma L\left(1, p^{d}\right)$ consisting of all transformations of the form $\xi \mapsto \alpha \xi^{t}$, where $\alpha$ is a nonzero element of the field and $t$ is an automorphism of the field. The group $\Gamma L\left(1, p^{d}\right)$ is metacyclic of order $\left(p^{d}-1\right) d$. A classification for nonsolvable groups has been carried out by Hering [5], 6]. It is considerably more complicated to state and prove, but among other things it shows that such groups have only a single nonsolvable composition factor (a summary is given in [8, page 386]).

Since the latter half of hypothesis $(*)$ is certainly satisfied in a solvable group, we can specialize to solvable groups and drop the condition that $\chi$ is faithful in order to obtain the following theorem.

Theorem 9. Let $G$ be a finite solvable group which has an irreducible character $\chi$ which takes the value 0 on only one conjugacy class $\mathcal{C}$. Let $K:=\langle\mathcal{C}\rangle$. Then:

(a) $K=G^{(k)}$ for some $k \geq 0$.

(b) There is a unique normal subgroup $L$ of $G$ such that $K / L$ is a chief factor of $G$ and $K \backslash L=\mathcal{C}$ (we set $|K: L|=p^{d}$ ).

(c) $G / K$ acts transitively on the set $(K / L)^{\#}$ of nontrivial elements of the vector space $K / L$ and so is one of the groups classified by Huppert.

(d) If $\chi$ is primitive, then $K / \operatorname{ker} \chi$ is an extra special group of order $p^{d+1}$ with centre $L /$ ker $\chi$.

(e) If $\chi$ is imprimitive, then $G / L$ is a 2-transitive Frobenius group of degree $p^{d}$.

Remark 10. We also note that (c) and Huppert's classification show that the integer $k$ in (a) is bounded. Indeed, since $\Gamma L\left(1, p^{d}\right)$ is metacyclic, $k=1$ or 2 except in the six exceptional cases. Computations using GAP [4] show that in the remaining cases $k \leq 4$.

Proof. (a) Let $k$ be the largest integer such that $K \leq G^{(k)}$. By Corollary 2 we know that the restriction $\chi_{G^{(k+1)}}$ is reducible, and so $G^{(k+1)} \leq L$. Therefore $G^{(k+1)} \leq L<K \leq G^{(k)}$, and so $G^{(k)} \leq C_{G}(K / L)$. Hence $K=G^{(k)}$ by Proposition 6 .

(b), (c) and (d) follow from Proposition 1, Corollary 7 and Proposition 4.

(e) Let $M$ be the subgroup defined in Proposition 5. Since $\chi$ is induced from a character on $M$, its restriction $\chi_{M}$ must be reducible, and so [1, page 145] shows that

$$
2 \leq\left[\chi_{M}, \chi_{M}\right] \leq 1+\frac{|\mathcal{C} \backslash M|}{|M|}=1+\frac{|\mathcal{C}|}{|M|} .
$$

Hence $|\mathcal{C}| \geq|M|$. Since $G=M K$ and $G / K$ acts transitively on $(K / L)^{\#}$ we conclude using Proposition 5 that

$$
\frac{|K|}{|L|}-1=p^{d}-1 \leq|G: K|=|M: M \cap K|=|M: L| \leq \frac{|\mathcal{C}|}{|L|} .
$$


However, $|\mathcal{C}| /|L|=p^{d}-1$ by Proposition 1] so equality must hold throughout. Thus $|M: L|=p^{d}-1$. Hence $M / L$ acts regularly on $(K / L)^{\#}$ and so $G / L=$ $(M / L)(K / L)$ is a 2 -transitive Frobenius group.

Remark 11. Not all groups having an irreducible character which takes 0 on a single conjugacy class satisfy the second half of hypothesis $(*)$. For example, the Atlas 3] shows that $A_{5}$ has three characters with this property and its central cover $2 \cdot A_{5}$ also has three. The group $L_{2}(7)$ has two characters with the required property, and each of the groups $L_{2}\left(2^{k}\right)(k=3,4, \ldots)$ appears to have one such character (of degree $2^{k}$ ). It would be interesting to know if these were the only simple groups with this property, or whether a group with such a character can have more than one nonabelian composition factor (see Remark 8). Another question which can be asked is what can be said about the kernel of such a character; evidently this kernel is contained in the normal subgroup $L_{0}:=\{u \in G \mid u \mathcal{C}=\mathcal{C}\}$.

\section{REFERENCES}

[1] Ya. G. Berkovich and E.M. Zhmud', Characters of Finite Groups. Part (2), Vol. 181, Mathematical Monographs, Amer. Math. Soc., Rhode Island, 1999. MR1650707 (99j:20007)

[2] D. Chillag, On zeros of characters of finite groups, Proc. Amer. Math. Soc. 127 (1999) 977-983. MR.1487363 (99f:20012)

[3] J.H. Conway et al., Atlas of Finite Simple Groups, Clarendon Press, Oxford, 1985. MR0827219 (88g:20025)

[4] The GAP Group, GAP-Groups, Algorithms and Programming, Version 4.4.4 (2005) (http://www.gap-system.org).

[5] Ch. Hering, Transitive linear groups and linear groups which contain irreducible subgroups of prime order, Geometriae Dedicata 2 (1974) 425-460. MR0335659 (49:439)

[6] Ch. Hering, Transitive linear groups and linear groups which contain irreducible subgroups of prime order II, J. Algebra 93 (1985) 151-164. MR0780488 (86k:20046)

[7] B. Huppert, Endliche Gruppen I, Springer-Verlag, Berlin, 1967. MR0224703 (37:302)

[8] B. Huppert and N. Blackburn, Finite Groups III, Springer-Verlag, Berlin, 1982. MR0662826 (84i:20001b)

[9] I.M. Isaacs, Character Theory of Finite Groups, Academic Press, New York, 1976. MR0460423 (57:417)

[10] I.M. Isaacs, Character degrees and derived length of a solvable group, Canad. J. Math. 27 (1975) 146-151. MR0357571(50:10039)

[11] E.M. Zhmud', On finite groups having an irreducible complex character with one class of zeros, Soviet Math. Dokl. 20 (1979) 795-797. MR0553828 (81g:20020)

School of Mathematics and Statistics, Carleton University, Ottawa, Ontario, CANADA K1S 5B6

Institute for Advanced Studies in Basic Sciences, Zanjan, P.O. Box 45195-1159, Iran - And - Islamic Azad University, Zanjan, P.O. Box 49195-467, Iran

E-mail address: rahnama@iasbs.ac.ir 